\title{
REVIEW ON ETHNOBOTANICAL PLANT SOURCES FOR MOSQUITO REPELLENCY USED BY TRIBES OF SOUTH INDIA
}

\author{
PRATHYUSHA KANTHETI ${ }^{1} \&$ SHALINI. $\mathbf{G}^{\mathbf{2}}$ \\ ${ }^{1}$ Research Scholar, Department of Apparel and Textiles, College of Home Science, \\ Professor Jayashankar Telangana State Agricultural University, Hyderabad, Telangana, India \\ ${ }^{2}$ Teaching Associate, Department of Apparel and Textiles, College of Home Science, \\ Professor Jayashankar Telangana State Agricultural University, Hyderabad, Telangana, India
}

\begin{abstract}
Mosquitoes have become a serious threat to public health, transmitting several dangerous diseases, especially in tropical regions. There has been a large increase in the insecticide resistance of this vector, and it has become a global problem. Carbon dioxide, lactic acid and many other odors released from the body of warm blooded animals are the major attracts of mosquitoes. Numbers of mosquito repellents are available in the market, both synthetic and natural. Depending on the type of phytochemical present, plant sources, acts as either repellent or as an insecticide for killing the larvae of mosquitoes. The repellent activity of plant sources has been explored by human beings for many years, and man started using these plant sources in different forms like burning of leaves, hanging the plant at the entrance, external application of oil from plant extracts or growing the mosquito repellent plants around the houses. The use of plant sources as active mosquito repellents was even recorded in the writings of Greek and Roman Scholars. In many parts of rural India and other parts of the world, plant based mosquito repellents are still in use. For eg: Plant sources, which have repellent activity, are preferred as a good and safe source by people in rural areas of Europe and North America. The plant sources, which were reviewed and found to be used as mosquito repellents by tribes of South India are Cymbopogon flexuosus, Ocimum americanum, Tinospora cordifolia (Willd.), Miers Pentanema indicum (Linn.), Ling Bixa Orellana L, eucalyptus globules and, Cassia fistula. These were used as topical application of leaf juice (or) essential oils, extracted from leaves and plant parts. Whereas, Adhatoda Vasica Euphorbia Milli Des. Azardiracta Indica A.Juss, Dalbergia sissoo DC, Vitex negundo Linn, Artimesia vulgaris L, Clausena anisata, Adhatoda Vasica and Anacardium Occidentale in different forms.
\end{abstract}

KEYWORDS: Mosquito Repellents, Natural Sources, Tribes of South India \& Medicinal Plants for Mosquito Repellency

Received: Apr 01, 2017; Accepted: Apr 27, 2017; Published: Jun 02, 2017; Paper Id.: IJMPSJUN20176

\section{INTRODUCTION}

There are over 3500 discovered mosquito species in the world; three most significant of these are the Aedes, Anopheles, and Culex. According to the World Health Organization (WHO), more than 1 million people die every year due to mosquito bites and the majority are due to malaria. A mosquito repellent can be in any form, i.e., it can be applied to the fabric, skin or any other surfaces in order to repel the host seeking mosquitoes. Many types of mosquito repellents are available in the market, both chemical and natural. For many years, the plants are considered as the valuable sources not only in preventing the mosquito bites, but also for many other medicinal values. Plants contain different types of bioactive chemicals which play an active role in preventing the attack of diverse kinds of insects. 
Different parts of the plants have been used in the traditional medications in tribal communities for several years. Globally 20,000 medicinal plants have been identified by the World Health Organization (WHO), Out of which, India's contribution is 15-20\%. In India, about 2000 drugs used are of plant origin. Tribes even depend on plant sources for mosquito repellent. Repellents play an important role in protecting man from different kinds of insects apart from mosquitoes. Burning of some herbs like Artemisia and Azadirachta indica produces smoke which has been used for the protection against mosquitoes and biting insects since ancient times. Planting of plant species like Azadirachta indica, Annona squamosa, Artemisia vulgaris, Cymbopogon citratus, Lantana camara, Ocimum sanctum and Vitex peduncular nearby the houses or settlement areas is another traditional method of controlling mosquito borne malaria, which is still in practice among many tribal communities in India. Still, in many rural areas of India, Polishing of house with extracts of different types of plants like Azadirachta, Artemisia, Lantana, Ocimum and Cymbopogon is routinely done in rural India to impel the mosquitoes and other flies.

\section{Phytochemicals}

Phytochemicals are the naturally occurring chemical compounds present in the plants, which mean Phyto-"plant" in Greek. The majority of the phytochemicals is accountable for colour and other phytochemicals are responsible in imparting the properties like, smell and flavour.

A unique biological activity is present in the phytochemicals derived from the plant sources. These phytochemicals derived from plant sources, acts as repellents, growth regulators and reproductive inhibitors.

Phytochemicals can be extracted from either whole plants or specific parts of the plant, depending on the activity of the derivatives. These bioactive chemicals occur in different parts of plants like roots, leaves, bark, flowers, stems, etc. Investigators have found that the effectiveness of chemicals derived from specific plant parts often varies with the mosquito species. Certain phytochemicals have photo-activated toxins that are reported effective against mosquitoes. Different phytochemicals in plants act or react with the mosquitoes in numerous ways for eg: few phytochemical acts as repellents, some acts as growth regulators, several acts as general toxicants to the life stages of mosquitoes, while few bioactive chemicals acts on the olfactory receptors.

\section{Mode of Action of Phytochemicals in Insect Body}

Plant extracts are generally known as the secondary metabolites that protect them from the herbivore. These metabolites are generally toxic, that are harmful for the insects and effect on the target molecules that are bio membranes, nucleic acid, cellular components and proteins, due to this, the insect physiology is disturbed that affect the nervous system such as synthesis of neurotransmitter, storage, release and activation of receptors. These phytochemicals act on the mosquito neurosystem in one or the other ways, like few inhibits the cellular respiration and potassium-sodium exchange. Few phytochemicals cause a nerve impulse, which blocks the nerve transmission in mosquitoes and insects.

\section{Tribes of India}

Plants/Herbs have been used by the tribal dwellings in remote places for many years, in meeting their lively hood and health care needs. These sources have been used by them in treating different kinds of diseases since time immemorial. Since there is increased interest in herbal products these days, even in modern or urban people, there is immense scope for research in validation of traditional claims for development of new drugs or medicines. 


\section{Review on Mosquito Repellent Plant Sources Used by Tribes of South India}

Here is the list of plant species used for generations by various tribes throughout south India in different forms.

Table 1: Plant Sources Used By Different Tribes of South India for Mosquito Repellency

\begin{tabular}{|c|c|c|c|c|c|c|}
\hline Sl. No. & State & Tribes & Plant Sources & Family & $\begin{array}{l}\text { Common } \\
\text { Name }\end{array}$ & Form of Usage \\
\hline \multirow{9}{*}{1.} & \multirow{4}{*}{$\begin{array}{l}\text { Tamil Nadu } \\
\text { (Arulappan et.al } \\
(2015) \text { ) }\end{array}$} & \multirow{2}{*}{ Siruvani hills } & $\begin{array}{l}\text { Cissampelos } \\
\text { pareira L., }\end{array}$ & Menispermaceae & Midwife's herb & $\begin{array}{l}\text { Topical } \\
\text { Application of } \\
\text { Leaf Juice }\end{array}$ \\
\hline & & & $\begin{array}{l}\text { Ocimum canum } \\
\text { Sims }\end{array}$ & Lamiaceae & Haory Basil & $\begin{array}{l}\text { The whole plant is } \\
\text { grown inside } \\
\text { house }\end{array}$ \\
\hline & & \multirow[t]{2}{*}{ Kalrayan Hill } & $\begin{array}{l}\text { Premna latifolia } \\
\text { Roxb }\end{array}$ & Verbenaceae & $\begin{array}{l}\text { Dusky fire } \\
\text { brand bark }\end{array}$ & $\begin{array}{l}\text { Dried stem soaked } \\
\text { in the water are } \\
\text { used as mosquito } \\
\text { repellant }\end{array}$ \\
\hline & & & $\begin{array}{l}\text { Azadirachta indica } \\
\text { A.Juss. }\end{array}$ & Meliaceae & Neem & $\begin{array}{l}\text { Burned fumes } \\
\text { from the leaves }\end{array}$ \\
\hline & \multirow{5}{*}{$\begin{array}{l}\text { Mustapha et.al } \\
(2015)\end{array}$} & \multirow{5}{*}{$\begin{array}{l}\text { Irulars tribes } \\
\text { from Siruvani } \\
\text { hills- nilgiri } \\
\text { mountains }\end{array}$} & $\begin{array}{l}\text { Aloe vera (L) } \\
\text { Burm.f }\end{array}$ & Liliaceae & Aloe vera & $\begin{array}{l}\text { Juice extracted } \\
\text { from plant is } \\
\text { sprayed in houses }\end{array}$ \\
\hline & & & $\begin{array}{l}\text { Anisomeles indica } \\
\text { (L.) Kuntze. }\end{array}$ & Lamiaceae & Catmint & $\begin{array}{l}\text { The dried leaves } \\
\text { are powdered and } \\
\text { sprayed as } \\
\text { sambrani (emits } \\
\text { fragrant fumes } \\
\text { when burnt) }\end{array}$ \\
\hline & & & $\begin{array}{l}\text { Azadirachta indica } \\
\text { A.Juss. }\end{array}$ & Meliaceae & Neem & $\begin{array}{l}\text { The leaves are } \\
\text { burnt and the } \\
\text { fumes act as a } \\
\text { repellent against } \\
\text { insects and } \\
\text { mosquito }\end{array}$ \\
\hline & & & Helicteres isora L. & Malvaceae & $\begin{array}{l}\text { Indian Screw } \\
\text { Tree }\end{array}$ & $\begin{array}{l}\text { Topicalapplication } \\
\text { of Juice extracted } \\
\text { from plant parts }\end{array}$ \\
\hline & & & $\begin{array}{l}\text { Ocimim } \\
\text { tenuiflorum L }\end{array}$ & Lamiaceae & $\begin{array}{l}\text { Holy } \\
\text { basil/Tulsi }\end{array}$ & $\begin{array}{l}\text { Topical } \\
\text { Application of } \\
\text { essential oils and } \\
\text { Juice extracted } \\
\text { from leaves } \\
\text { applied to skin. }\end{array}$ \\
\hline & Senthil et.al (2015) & $\begin{array}{l}\text { Kalrayan hill, } \\
\text { Eastern Ghats of } \\
\text { Tamilnadu }\end{array}$ & $\begin{array}{l}\text { Premna latifolia } \\
\text { Roxb }\end{array}$ & Verbenaceae & $\begin{array}{l}\text { Dusky fire } \\
\text { brand bark }\end{array}$ & $\begin{array}{l}\text { Dried stem soaked } \\
\text { in the water are } \\
\text { used as mosquito } \\
\text { repellant }\end{array}$ \\
\hline & \multirow[b]{2}{*}{ Karthik et.al (2014) } & \multirow[b]{2}{*}{$\begin{array}{l}\text { Paliyar Tribes of } \\
\text { Sembarankulam, } \\
\text { Dindigul District }\end{array}$} & $\begin{array}{l}\text { Azadirachta indica } \\
\text { A.Juss. }\end{array}$ & Meliaceae & Neem & $\begin{array}{l}\text { Burnt Fumes of } \\
\text { Dried leaves }\end{array}$ \\
\hline & & & Ficus Hispida L. & $\begin{array}{l}\text { Moraceae (Mulberry } \\
\text { family) }\end{array}$ & Hairy Fig & $\begin{array}{l}\text { Placement of } \\
\text { crushed fruits near } \\
\text { mosquito affected } \\
\text { area acts as } \\
\text { mosquito } \\
\text { repellent. }\end{array}$ \\
\hline & $\begin{array}{l}\text { Vijayalakshmi et.al } \\
\text { (2011) }\end{array}$ & $\begin{array}{l}\text { Cuddalore } \\
\text { district, } \\
\text { Tamilnadu } \\
\end{array}$ & $\begin{array}{l}\text { Azadirachta indica } \\
\text { A.Juss. }\end{array}$ & Meliaceae & Neem & $\begin{array}{l}\text { Burnt Fumes of } \\
\text { Dried leaves }\end{array}$ \\
\hline & \multirow{2}{*}{$\begin{array}{l}\text { Subramanyam et.al } \\
(2009)\end{array}$} & \multirow{2}{*}{$\begin{array}{l}\text { Irulas tribe in the } \\
\text { Kodiakkarai } \\
\text { Reserve Forest }\end{array}$} & $\begin{array}{l}\text { Azadirachta } \\
\text { indica A. Juss }\end{array}$ & Meliaceae & Neem & $\begin{array}{l}\text { Burnt Fumes of } \\
\text { Dried leaves with } \\
\text { charcoal. }\end{array}$ \\
\hline & & & Vitex negundo L. & Verbenaceae & Chaste Tree & $\begin{array}{l}\text { Topical } \\
\text { application of leaf } \\
\text { extracts }\end{array}$ \\
\hline & $\begin{array}{l}\text { Ayyanar et.al } \\
(2005)\end{array}$ & $\begin{array}{l}\text { Kani tribals in } \\
\text { Kouthalai of } \\
\text { Tirunelveli hills }\end{array}$ & $\begin{array}{l}\text { Cipadessa } \\
\text { baccifera Miq + } \\
\text { Vitex negundo }\end{array}$ & $\begin{array}{l}\text { Meliaceae+ } \\
\text { Verbenaceae }\end{array}$ & $\begin{array}{l}\text { Maramalli+ } \\
\text { Chaste Tree }\end{array}$ & $\begin{array}{l}\text { Topical } \\
\text { Application of } \\
\text { juice extracted } \\
\text { with mixture of } \\
\text { these two leaves. }\end{array}$ \\
\hline 2. & $\begin{array}{l}\text { Kerala } \\
\text { (Khan et.al (2016)) }\end{array}$ & Attappadi & $\begin{array}{l}\text { Cymbopogon } \\
\text { Citratus (L.) } \\
\end{array}$ & Poaceae & Lemon Grass & $\begin{array}{l}\text { Application of } \\
\text { Leaf extracts on }\end{array}$ \\
\hline
\end{tabular}




\begin{tabular}{|c|c|c|c|c|c|c|}
\hline & & & & & & $\begin{array}{l}\text { external body } \\
\text { parts }\end{array}$ \\
\hline & & & Murraya koenigii & Rutaceace & Curry leaf & $\begin{array}{l}\text { Topical } \\
\text { application of } \\
\text { essential oils } \\
\text { extracted from } \\
\text { leaves }\end{array}$ \\
\hline & & & $\begin{array}{l}\text { Ricinus communis } \\
\text { (L.) }\end{array}$ & Euphorbiaceae & $\begin{array}{l}\text { Castor bean } \\
\text { plant }\end{array}$ & $\begin{array}{l}\text { Fumes of leaves } \\
\text { when burned } \\
\text { along with } \\
\text { charcoal acts as } \\
\text { mosquito repellent }\end{array}$ \\
\hline & (Haridas et.al & Kattunavakans & $\begin{array}{l}\text { Pandanus } \\
\text { odoratissimus L.f. }\end{array}$ & Arecaceae & Kewda & $\begin{array}{l}\text { Crushed } \\
\text { inflorescences are } \\
\text { mixed with water } \\
\text { and sprayed over } \\
\text { mosquito affected } \\
\text { areas }\end{array}$ \\
\hline & $(2015))$ & Kattunayakans & $\begin{array}{l}\text { Pongamia pinnata } \\
\text { (L.) Pierre }\end{array}$ & Fabaceae & Indian Beech & $\begin{array}{l}\text { Crushed leaves } \\
\text { and barks are } \\
\text { mixed with water } \\
\text { and sprayed over } \\
\text { mosquito affected } \\
\text { areas. }\end{array}$ \\
\hline & Jose et.al ( 2015) & $\begin{array}{l}\text { Paniayas tribe of } \\
\text { wayanad district }\end{array}$ & Leucas Zeylanica & Lamiaceae & Thumba & $\begin{array}{l}\text { Topical } \\
\text { application of } \\
\text { essential oil } \\
\text { derived from } \\
\text { whole plant }\end{array}$ \\
\hline & Limcy et.al (2013) & $\begin{array}{l}\text { Attappady and } \\
\text { Vakkodan Hill } \\
\text { region- } \\
\text { Jellippara, Agaly, } \\
\text { Kottathara and } \\
\text { Kookampalayam }\end{array}$ & Butea monosperma & Fabaceae & $\begin{array}{l}\text { Flame of the } \\
\text { forest }\end{array}$ & $\begin{array}{l}\text { Gum like juice } \\
\text { from its flower } \\
\text { kills mosquito }\end{array}$ \\
\hline & & & $\begin{array}{l}\text { Canarium strictum } \\
\text { added with Allium } \\
\text { sativum } \\
\text { and Curcuma } \\
\text { aromatic. }\end{array}$ & $\begin{array}{l}\text { Burseraceae+Alliaceae } \\
+ \text { Zingiberaceae }\end{array}$ & $\begin{array}{l}\text { Black } \\
\text { Dammar+ } \\
\text { Garlic+Wild } \\
\text { Turmeric }\end{array}$ & $\begin{array}{l}\text { Infusion of all the } \\
\text { three in powder } \\
\text { form and using } \\
\text { them as Topical } \\
\text { application }\end{array}$ \\
\hline & $\begin{array}{l}\text { Karnataka } \\
\text { ((Venkatachalapathi } \\
\text { et.al (2016)) }\end{array}$ & Irula tribes & $\begin{array}{l}\text { Canarium } \\
\text { strictum Roxb. }\end{array}$ & Burseraceae & $\begin{array}{l}\text { Black } \\
\text { Dammar }\end{array}$ & $\begin{array}{l}\text { Topical } \\
\text { application of } \\
\text { Bark Powder }\end{array}$ \\
\hline & & & $\begin{array}{l}\text { Melia dubia added } \\
\text { with Allium } \\
\text { sativum, Canarium } \\
\text { strictum } \\
\text { and Curcuma } \\
\text { aromatic. }\end{array}$ & $\begin{array}{l}\text { Meliaceae + } \\
\text { Alliaceae+ } \\
\text { Burseraceae+ } \\
\text { Zingiberaceae }\end{array}$ & $\begin{array}{l}\text { Malabar } \\
\text { Neem+Garlic } \\
+ \text { Black } \\
\text { Dammar+Wild } \\
\text { Turmeric }\end{array}$ & $\begin{array}{l}\text { Topical } \\
\text { application of } \\
\text { mixture of four } \\
\text { powders }\end{array}$ \\
\hline & & & Melia dubia L. & Meliaceae & Malabar Neem & $\begin{array}{l}\text { Topical } \\
\text { application of } \\
\text { Bark Powder }\end{array}$ \\
\hline 3. & $\begin{array}{l}\text { (Prakasha et.al } \\
(2006))\end{array}$ & $\begin{array}{l}\text { Sringeri taluk ( } \\
\text { Kavadi, } \\
\text { Kuthugodu, } \\
\text { Nemmaru Kigoa }\end{array}$ & $\begin{array}{l}\text { Adhatoda } \\
\text { vasica Nees. }\end{array}$ & Acanthaceae & Malabar nut & $\begin{array}{l}\text { The plant is burnt } \\
\text { and the fumes are } \\
\text { used as mosquito } \\
\text { and pest } \\
\text { repellents. }\end{array}$ \\
\hline & & and Hulugaru) & Vitexnegundo Linn & Verbenaceae & Chaste Tree & $\begin{array}{l}\text { Topical } \\
\text { application of leaf } \\
\text { Extracts }\end{array}$ \\
\hline & $\begin{array}{l}\text { Natarajan et.al } \\
(2003)\end{array}$ & $\begin{array}{l}\text { Bidar, Gulbarga, } \\
\text { Raichur, Yadgir }\end{array}$ & $\begin{array}{l}\text { Vitexneugundo } \\
\text { Linn }\end{array}$ & Verbenaceae & Chaste Tree & $\begin{array}{l}\text { Topical } \\
\text { application of fruit } \\
\text { pulp }\end{array}$ \\
\hline & & $\begin{array}{l}\text { Mysore and Coor } \\
\text { districts- Tribes } \\
\text { namely- }\end{array}$ & $\begin{array}{l}\text { Aloe Vera (L) } \\
\text { Burm f. }\end{array}$ & Liliaceae & Aloe Vera & $\begin{array}{l}\text { Growing of plant } \\
\text { in and around } \\
\text { house }\end{array}$ \\
\hline & $\begin{array}{l}\text { Kshirsagar et.al } \\
(2001)\end{array}$ & $\begin{array}{l}\text { jenukuruba } \\
\text { bettakuruba. } \\
\text { Soliga, yerava, } \\
\text { Panjariyerava, } \\
\text { malekudia, } \\
\text { Tammadi medha, } \\
\text { hakki-pikki, }\end{array}$ & $\begin{array}{l}\text { Pongamia pinnata } \\
\text { (L) Pierre }\end{array}$ & Fabaceae & Indian beech & $\begin{array}{l}\text { Seed oil is mixed } \\
\text { with kerosene to } \\
\text { light up the lamps }\end{array}$ \\
\hline
\end{tabular}




\begin{tabular}{|c|c|c|c|c|c|c|}
\hline & & $\begin{array}{l}\text { paniyerava } \\
\text { gowdakuruba, } \\
\text { kadu-kuruba, } \\
\text { kaniyan and } \\
\text { girijana }\end{array}$ & & & & \\
\hline \multirow{14}{*}{4.} & \multirow{4}{*}{$\begin{array}{l}\text { Andhra Pradesh } \\
\text { and Telangana } \\
\text { (Parijatam et.al } \\
((2016))\end{array}$} & \multirow{4}{*}{$\begin{array}{l}\text { Jatapus, } \\
\text { Kondadoras, } \\
\text { Mukadoras, } \\
\text { Mannedoras, } \\
\text { Yerukulas, } \\
\text { Goudus, Gadabas } \\
\text { and Savara. }\end{array}$} & $\begin{array}{l}\text { Acacia nilotica } \\
\text { (Linn.) Willd }\end{array}$ & Mimosaceae & Gum Arabic & $\begin{array}{l}\text { Essential oil } \\
\text { extracted from } \\
\text { stem bark is } \\
\text { applied on } \\
\text { external body } \\
\text { parts to repel } \\
\text { mosquitoes }\end{array}$ \\
\hline & & & Cassia alata Linn. & Caesalpinaceae & Candle Bush & $\begin{array}{l}\text { Dried and fresh } \\
\text { leaves are burned } \\
\text { and the fumes acts } \\
\text { as mosquito } \\
\text { repellent. }\end{array}$ \\
\hline & & & $\begin{array}{l}\text { Cymbopogon } \\
\text { flexuosus (Linn.) } \\
\text { Rendie. }\end{array}$ & Poaceae & Malabar Grass & $\begin{array}{l}\text { Oil acts as an } \\
\text { excellent repellent } \\
\text { against } \\
\text { mosquitoes for } \\
\text { hours. }\end{array}$ \\
\hline & & & $\begin{array}{l}\text { Pongamia pinnata } \\
\text { (Linn.) Pierre }\end{array}$ & Leguminoseae & Indian beech & $\begin{array}{l}\text { Seed oil applied } \\
\text { on external parts } \\
\text { of body }\end{array}$ \\
\hline & \multirow{2}{*}{$\begin{array}{l}\text { Rajgopal et.al } \\
(2015)\end{array}$} & \multirow{2}{*}{$\begin{array}{l}\text { Lankamalleswara } \\
\text { wildlife } \\
\text { sanctuary, } \\
\text { Kadapa District }\end{array}$} & $\begin{array}{l}\text { Chloroxylon } \\
\text { swietenia DC }\end{array}$ & Flindersiaceae & $\begin{array}{l}\text { East India } \\
\text { Satin Wood }\end{array}$ & $\begin{array}{l}\text { Stem bark paste } \\
\text { made into bolls } \\
\text { and used as } \\
\text { mosquito repellent }\end{array}$ \\
\hline & & & $\begin{array}{l}\text { Tinospora } \\
\text { cordifolia (Willd.) } \\
\text { Miers }\end{array}$ & Menispermaceae & $\begin{array}{l}\text { Heart- leaved } \\
\text { moon seed }\end{array}$ & $\begin{array}{l}\text { Leaf paste made } \\
\text { into bolls and } \\
\text { used as Mosquito } \\
\text { repellent }\end{array}$ \\
\hline & \multirow{3}{*}{$\begin{array}{l}\text { (Saidulu et.al } \\
(2015))\end{array}$} & \multirow{3}{*}{$\begin{array}{l}\text { Pocharam } \\
\text { wildlife } \\
\text { Sanctuary, } \\
\text { Telangana }\end{array}$} & $\begin{array}{l}\text { Chloroxylon } \\
\text { swietenia }\end{array}$ & Rutaceae & $\begin{array}{l}\text { East India } \\
\text { Satin wood }\end{array}$ & $\begin{array}{l}\text { Fumigants of the } \\
\text { fresh/dried } \\
\text { wood/leaf acts as } \\
\text { mosquito repellen }\end{array}$ \\
\hline & & & Hyptis suaveolens & Lamiaceae & $\begin{array}{l}\text { American } \\
\text { Mint }\end{array}$ & $\begin{array}{l}\text { The dried plant } \\
\text { parts are burned } \\
\text { and the fumes acts } \\
\text { as mosquito } \\
\text { repellents }\end{array}$ \\
\hline & & & $\begin{array}{l}\text { Ocimum } \\
\text { americanum }\end{array}$ & Lamiaceae & Spice Basil & $\begin{array}{l}\text { The juice } \\
\text { extracted gives a } \\
\text { sweet scent of } \\
\text { camphor which } \\
\text { when applied on } \\
\text { body helps in } \\
\text { repelling } \\
\text { mosquitoes }\end{array}$ \\
\hline & $\begin{array}{l}\text { Bharath Kumar } \\
\text { et.al (2014) }\end{array}$ & $\begin{array}{l}\text { Tribals of } \\
\text { Sriharikota } \\
\text { Island, Andhra } \\
\text { Pradesh- } \\
\text { Penubakam, } \\
\text { Kothachenu and } \\
\text { Chengalpalem }\end{array}$ & $\begin{array}{l}\text { Eugenia bracteata } \\
\text { (Willd.) DC }\end{array}$ & Myrtaceae & $\begin{array}{l}\text { Shining } \\
\text { Bracteata }\end{array}$ & $\begin{array}{l}\text { Fresh leaves taken } \\
\text { and burnt. The } \\
\text { smoke acts as } \\
\text { mosquito } \\
\text { repellent. }\end{array}$ \\
\hline & \multirow[b]{2}{*}{ Reddy et.al (2014) } & \multirow[b]{2}{*}{$\begin{array}{l}\text { Medak district, } \\
\text { Andhra Pradesh- }\end{array}$} & $\begin{array}{l}\text { Pentanema } \\
\text { indicum (Linn.) } \\
\text { Ling }\end{array}$ & Asteraceae & Sonkadi & $\begin{array}{l}\text { Leaf juice used as } \\
\text { a lotion to repel } \\
\text { mosquitoes. }\end{array}$ \\
\hline & & & $\begin{array}{l}\text { Premna latifolia } \\
\text { Roxb. }\end{array}$ & Verbenaceae & $\begin{array}{l}\text { Dusky Fire } \\
\text { Brand Bar }\end{array}$ & $\begin{array}{l}\text { The twigs are } \\
\text { burnt and fumes } \\
\text { spread out in the } \\
\text { home/ dried stem } \\
\text { soaked in the } \\
\text { water }\end{array}$ \\
\hline & \multirow[t]{2}{*}{ Padal et.al (2013) } & \multirow{2}{*}{$\begin{array}{l}\text { Arakuvalley } \\
\text { Mandalam, } \\
\text { Visakhapatnam } \\
\text { District }\end{array}$} & $\begin{array}{l}\text { Chloroxylon } \\
\text { swietenia DC }\end{array}$ & Flindersiaceae & $\begin{array}{l}\text { Ceylon satin } \\
\text { wood }\end{array}$ & $\begin{array}{l}\text { Stem bark paste } \\
\text { made into bolls } \\
\text { and used as } \\
\text { mosquito repellent }\end{array}$ \\
\hline & & & $\begin{array}{l}\text { Cymbopogon } \\
\text { flexuosus (Linn.) } \\
\text { Rendle. }\end{array}$ & Poaceae & Lemon grasss & $\begin{array}{l}\text { Leaf juice is } \\
\text { sprayed around } \\
\text { the surroundings }\end{array}$ \\
\hline
\end{tabular}




\begin{tabular}{|c|c|c|c|c|c|c|}
\hline & & & & & & $\begin{array}{l}\text { of the house for } \\
\text { mosquito repellent }\end{array}$ \\
\hline & $\begin{array}{l}\text { Ravi Prasad et.al } \\
\text { (2011) }\end{array}$ & $\begin{array}{l}\text { Rudrakod Sacred } \\
\text { Grove in } \\
\text { Nallamalais- } \\
\text { Chenchu Tribes }\end{array}$ & $\begin{array}{l}\text { Chloroxylon } \\
\text { swietenia DC }\end{array}$ & Rutaceae & $\begin{array}{l}\text { Ceylon satin } \\
\text { wood }\end{array}$ & $\begin{array}{l}\text { The smoke of } \\
\text { burnt leave is used } \\
\text { as mosquito } \\
\text { repellent. }\end{array}$ \\
\hline & \multirow{3}{*}{ Pratap et.al (2009) } & \multirow{3}{*}{$\begin{array}{l}\text { Kailasagiri forest } \\
\text { range of Chittoor } \\
\text { district }\end{array}$} & $\begin{array}{l}\text { Anisomeles } \\
\text { malabarica (L.) }\end{array}$ & Lamiaceae & $\begin{array}{l}\text { Malabar } \\
\text { Catmint }\end{array}$ & $\begin{array}{l}\text { Small branches } \\
\text { are hung over at } \\
\text { entrance door to } \\
\text { drive out the } \\
\text { mosquitoes. }\end{array}$ \\
\hline & & & Bixa orellana L. & Bixaceae & Annatto & $\begin{array}{l}\text { External } \\
\text { application of } \\
\text { seed pulp is } \\
\text { indicated as } \\
\text { mosquito } \\
\text { repellant. }\end{array}$ \\
\hline & & & $\begin{array}{l}\text { Leucas aspera } \\
\text { (Willd.) Link }\end{array}$ & Lamiaceae & $\begin{array}{l}\text { Common } \\
\text { Leucas }\end{array}$ & $\begin{array}{l}\text { Small branches } \\
\text { are used as } \\
\text { festoons to drive } \\
\text { out the } \\
\text { mosquitoes }\end{array}$ \\
\hline \multirow{10}{*}{5.} & \multirow{7}{*}{$\begin{array}{l}\text { Orissa } \\
\text { (Pattanayak et.al ( } \\
\text { July 2015)) }\end{array}$} & \multirow{7}{*}{$\begin{array}{l}\text { Santal, Kolha, } \\
\text { Munda, Saora, } \\
\text { Shabar and } \\
\text { Bhottada, } \\
\text { Bhumij, Bhuiya, } \\
\text { Oraon, Paroja } \\
\text { and Kisan }\end{array}$} & $\begin{array}{l}\text { Ageratum } \\
\text { conyzoides L. }\end{array}$ & Asteraceae & $\begin{array}{l}\text { Billy goat } \\
\text { weed }\end{array}$ & $\begin{array}{l}\text { Dry } \\
\text { Leaves+cowdung }\end{array}$ \\
\hline & & & $\begin{array}{l}\text { Annona squamosa } \\
\mathrm{L}\end{array}$ & Annonaceae & Custard apple & Dry Leaves \\
\hline & & & $\begin{array}{l}\text { Barleria prionitis } \\
\text { L. }\end{array}$ & Acanthaceae & $\begin{array}{l}\text { Porcupine } \\
\text { flower }\end{array}$ & $\begin{array}{l}\text { Dry Leaves+ coir } \\
\text { pith }\end{array}$ \\
\hline & & & $\begin{array}{l}\text { Cellastrus } \\
\text { paniculata Willd }\end{array}$ & Celastraceae & $\begin{array}{l}\text { Intellect tree, } \\
\text { Black oil plant }\end{array}$ & Seed oil \\
\hline & & & $\begin{array}{l}\text { Eucalyptus } \\
\text { citriodora Hook }\end{array}$ & Myrtaceae & $\begin{array}{l}\text { Lemon } \\
\text { eucalyptus }\end{array}$ & $\begin{array}{l}\text { Dryleaves + } \\
\text { cowdung }\end{array}$ \\
\hline & & & Lantana camara L & Verbenaceae & Lantana & $\begin{array}{l}\text { Dry leaves+ } \\
\text { cowdung }\end{array}$ \\
\hline & & & $\begin{array}{l}\text { Trychnos nux } \\
\text { vomica L }\end{array}$ & Strychnaceae & Poison Nut & Seed oil \\
\hline & & & $\begin{array}{l}\text { Celastrus } \\
\text { paniculatus Wild }\end{array}$ & Celastraceae & $\begin{array}{l}\text { Black seed oil } \\
\text { plant }\end{array}$ & $\begin{array}{l}\text { Oil from seeds is } \\
\text { used as mosquito } \\
\text { repellent, leeches } \\
\text { and other biting } \\
\text { insects. }\end{array}$ \\
\hline & Panda et.al (2011) & district, Orissa & $\begin{array}{l}\text { Cymbopogon } \\
\text { martini (Roxb.) }\end{array}$ & Poaceae & Ginger Grass & $\begin{array}{l}\text { The Leaves are } \\
\text { crushed and the } \\
\text { juice extracted is } \\
\text { applied externally } \\
\text { on exposed body } \\
\text { parts. }\end{array}$ \\
\hline & Sahu et.al (2013) & $\begin{array}{l}\text { Boudh district of } \\
\text { Odhisha }\end{array}$ & $\begin{array}{l}\text { Diospyros } \\
\text { melanoxylon Roxb }\end{array}$ & Ebenaceae & Black Ebony & $\begin{array}{l}\text { Oil from seeds is } \\
\text { used as mosquito } \\
\text { repellent, leeches } \\
\text { and other biting } \\
\text { insects. }\end{array}$ \\
\hline & & & Adhatoda Vasica & Acanthaceae & Malabar nut & $\begin{array}{l}\text { Fumigants from } \\
\text { burned bark, } \\
\text { leaves and root } \\
\text { acts as Mosquito } \\
\text { repellent }\end{array}$ \\
\hline 6. & $\begin{array}{l}\text { Goa } \\
\text { (Satyawam Naik, } \\
\text { et.al (2014) }\end{array}$ & $\begin{array}{l}\text { Gawde, } \\
\text { Chambhar, mhar, } \\
\text { kansar }\end{array}$ & Cassia fistula, linn & Leguminosae & $\begin{array}{l}\text { Golden } \\
\text { shower }\end{array}$ & $\begin{array}{l}\text { Application of } \\
\text { leaf extracts on } \\
\text { the external body } \\
\text { parts. }\end{array}$ \\
\hline & & & $\begin{array}{l}\text { Eucalyptis } \\
\text { globules, labill }\end{array}$ & Myrtaceae & $\begin{array}{l}\text { Blue Gum } \\
\text { Tree }\end{array}$ & $\begin{array}{l}\text { Application of } \\
\text { leaf extracts on } \\
\text { the external body } \\
\text { parts. }\end{array}$ \\
\hline
\end{tabular}




\section{CONCLUSIONS}

The plant sources, which were found to be used as mosquito repellents by tribes are Cymbopogon flexuosus, Ocimum americanum, Tinospora cordifolia (Willd.), Miers Pentanema indicum (Linn.), Ling Bixa Orellana L, eucalyptus globules, Cassia fistula. These were used as topical application of leaf juice (or) essential oils extracted from leaves and plant parts. Whereas, Adhatoda Vasica Euphorbia Milli Des. Azardiracta Indica A. Juss, Dalbergia sissoo DC, Vitex negundo Linn, Artimesia vulgaris L, Clausena anisata, Adhatoda Vasica Anacardium Occidentale are used in the burnt form, i.e., fumigants from the burnt plant parts are used as mosquito repellents.

It is observed from this review conducted that burning of dried leaves from Azadirachta indica A.Juss, Vitex negundo L, various species of Ocimum, Adhatoda Vasica, Pongamia pinnata (Linn.) pierre, topical application of Aloe Vera (L) Burm f., Vitex negundo L., Ocimim tenuiflorum L., Ocimum canum Sims, Ocimum gratissimum Linn, Eucalyptis globules, labill and Lantana Camara in juice form or as essential oils extracted from leaves/plants, and planting of Azadirachta indica A.Juss and Ocimum sanctum $\mathrm{L}$ in and around the houses are the major ethno botanical sources to repel mosquitoes.

\section{ACKNOWLEDGEMENTS}

Sincerely convey my thanks to all the authors for providing an opportunity to write a review article on mosquito repellent plants sources used by different tribal communities of south India.

\section{REFRENCES}

1. Ayyanar, M., and Ignacimuthu, S., (2005). Traditional knowledge of Kani tribals in Kouthalai of Tirunelveli hills, Tamil Nadu, India. Journal of Ethnopharmacology.102:246-255.

2. Bharath Kumar, R., and Suryanarayana, B., (2014). Ethnomedicinal recipes for Ophthalmic and ENT problems \& allied diseases from Tribals of Sriharikota Island, Andhra Pradesh. Research Journal of Pharmaceutical, Biological and Chemical Sciences. 5(1): 853-871.

3. Haridas, R., Thangapandian, V., and Thomas, B., (2015). Ethnomedicinal Knowledge of Tribe-Kattunayakans in Nilambur Forests of Malappuram District, Kerala, India. International Journal of Phytotherapy. 5(2):76-85.

4. Jose et.al (2015) Jose, T., and Sebastian, A., (Oct 2015). Ethnobotanical Study of Traditional Medicinal Plants Used by Indigenous People in North Kerala. Indian Journal of Applied Research. 5(10):184-186.

5. Karthik, K., Parimelazhagan, T, Rajendran, A., and Thomas, B., (2014). Medico-Potential Plants Used by Paliyar Tribes of Sembarankulam, Dindigul District, Tamil Nadu, India. International Journal of Pharmacology Research. 4(2):69-74.

6. Khan, I., Rehman, A., Awan, A, S., and Shabbir, M., (2016). Importance of Ethnomedicinal Flora of Arian and Batwal Tribes of Punjab, India. Journal of Medicinal and Aromatic Plants.3(5): 17-23.

7. Kshirsagar, R, D., Singh, N, P.,(2001). Some Less Known Ethnomedicinal uses from Mysore and coorg districts, Karnataka, Southern India. Ancient Science of Life. 20:20-25.

8. Limcy, T, J., and Shubashini, K., (2013). Ethno Medicinal Practices of Tribal Inhabitants of Attappady and Vakkodan Hill Regions of Kerala. International Journal of Scientific Research. 2(3):33-36.

9. Padal et.al (2013) Padal, S, B., and Sandhyasri, B., (2013). Traditional use of Monocotyledon Plants of Arakuvalley Mandalam, Visakhapatnam District, Andhra Pradesh, India. IOSR Journal of Pharmacy and Biological Sciences. 6(2): 12-16. 
10. Panda, S,K., Rout, S, D., Mishra, N and Panda, T., (2011). Phytotherapy and traditional knowledge of tribal communities of Mayurbhanj district, Orissa, India. Journal of pharmacognosy and Phytotherapy.3(7): 101-113.

11. Parijatam, T, R., Sujatha, B., and Seetha Lakshmi, B., (2016). Ethnomedicinal studies of medicinal plants in Eastern ghats of Vizianagaram district, Andhra Pradesh, India. International Journal of Bioassays. 5(2):4825-4842

12. Prakasha et.al (2006)) Prakasha, H, M., and Krishnappa, M., (2006). People's knowledge on medicinal plants in Sringeri taluk, Karnataka. Indian Journal of Traditional Knowledge. 5(3): 353-357.

13. Pratap, G, P., Prasad, G, P., and Sudarsanam,G., (2009). Ethno Medical studies in Kailasagirikonda Forest Range of Chittoor District, Andhra Pradesh. Ancient Science of Life. 29 (2):40-45.

14. Rajagopal, S., Madhusudhana, A., and Suresh Babu., (2015). Traditional Medicinal Plants of Lankamalleswara Wildlife Sanctuary, Kadapa District, Andhra Pradesh, India. American Journal of Ethnomedicine. 2(6):379-391.

15. Ravi Prasad, B., and Sunitha, S., (2011). Medicinal Plant Resources of Rudrakod Sacred Grove in Nallamalais, Andhra Pradesh, India. Journal of Biodiversity. 2(2):75-89.

16. Sahu, C, R., Nayak, R, K., and Dhal, N, K., (2013). The plant wealth of Boudh district of Odisha, India with reference to Ethnobotany. International Journal of CurrentBiotechnology.

17. Saidulu, P., Satheesh, S., Ramesh, K., and Ragan, A., (2015). Ethnobotanical Knowledge Studied in Pocharam Wildlife Sanctuary, Telangana, India. Notulae Scientia Biologicae.(Academic press). 7(2):164-170.

18. Satyawan Naik., Puttaiah, E, T., and Ananth Nag, B., (2014). Ethonobotanical Studies of some Plants included in Folk Medicines of Goa. International Journal of Basic and Applied Science. 3(1): 6-13.

19. Subramanyam, R., and Newmaster, S, G., (2009). Valorizing the 'Irulas' traditional knowledge of medicinal plants in the Kodiakkarai Reserve Forest, India. Journal of Ethnobiology and Ethnomedicine. 5(10): 1-13.

20. Vijayalakshmi, R., and Ranganathan, R., (2011). Ethnobotanical Studies on Some Traditional Medicinal Plants in Cuddalore District. Journal of Drug Invention Today.3(7):160-164 